\title{
Dawn of viral epidemic treatment: the medical system based on body states control
}

\begin{abstract}
Year after year, the haze of flu keeps coming back. Dealing with the aggressive threat of virus, modern medicine appears to stand by helplessly. Virus mutates in an extraordinary manner and speed, it keeps frustrating the fantasy to control epidemics by means of vaccines. Cruel reality forces us to rethink the idea of killing virus to be considered as the only way to deal with viral epidemics. This article based on an in-depth analysis of the viral infectious diseases development mechanism, to propose a new viral epidemics treatment idea that aim at body states control. With this new idea, we do not need to find the exact pathogenic factor that caused infectious diseases, and we also do not need to find the way to kill the virus, but we can find an effective treatment for viral diseases. The therapeutic idea of treating body reactive states in response to virus infection as controllable variables, it is the internal mechanism that the Traditional Chinese Medicine (TCM)- the medical system that is irrelevant to the modern science - has been effectively deal with acute infectious diseases. To introduce the Syndrome Differentiation and Treatment System of TCM, and making it constantly precise by means of modern detection technology, artificial intelligence and big data analysis techniques. It is the shortcut for modern medicine to find the treatment of viral infectious diseases toward precision medicine era.
\end{abstract}

Keywords: complexity science, precision medicine, influenza, viral disease, body status medical
Volume 5 Issue 6 - 2017

Bing Yuan

Hong Kong Modern Chinese Medicine R \& D Center

Correspondence: Bing Yuan, Hong Kong Eastern Medicine Research Centre, Flat A-B, I 2/F, Bonds Mansion, 554-556 Nathan Road, Kowloon, Hong Kong, Tel +852 2300 1898, Email greenisland@vip.163.com

Received: January 24, 2017 | Published: March 16, 2017

\section{Introduction}

No matter it is SARS, bird flu, H1N1, or Ebola, year after year, humanity still cannot get rid of the virus haze. Faced with the spread of the virus, people as usual, put their hopes in the vaccine research. However, manufactured vaccine and epidemic viruses cannot be a hundred percent fit. The development of new vaccines cannot keep up with a much faster mutation rate of the virus. People are repeatedly frustrated by relying on the illusion of a vaccine to control the epidemic.

In a highly scientifically advanced and developed era like now, is there really nothing that we can do to combat virus but to administer Interferon or Tamiflu that can hardly cure but bring about a lot of undesirable adverse effects and leaving us perish in epidemics?

Forsaking traditional wisdom and forbidding future knowledge in the name of 'Science'. The modern medical system, based on scientific observation and experiments, we called today was developed $300 y e a r s$ ago. It excluded the connotation of ancient Greek conjecture. Thus, science evolved and developed on the basis of observations and experiments with the main characteristic "to change variable one at a time while the rest of the variables remain unchanged'. This single factor analytical method, after a few hundred years, formed today's enormous medical system from basic theory to clinical medicine with tireless efforts.

While the knowledge of modern medicine being built, an ingrained opinion has formed that observation and single factor analysis become the only scientific research method. Other methods are thrown away because they are considered as 'pseudo-scientific'. Therefore, scientists and researchers so trained would forsake methods that are considered 'pseudo-science'. And they would not deploy any research methods that they viewed upon as 'pseudo-science'. This explained why though we now are so helpless in front of epidemic yet modern medicine will not turn to TCM for help. As if this over a thousand years, TCM clinical experiences in the combat of epidemics and contagious diseases have not ever existed at all!

In the field of life science, the knowledge about human body, diseases and drugs are growing rapidly as the progresses in biology, physiology and medicine. However, because of the limitation of physiological factors, the percentage of knowledge grasped by one person is limited. For example: it the past, we named Dr. Zhang a medical expert, Dr. Li a surgeon, today we may refine it to cardiovascular expert and thoracic expert respectively. How do we call these specialist practitioners tomorrow?

The development of science leads our vision to a depth of specific field, but this depth results less understanding of the whole picture. Researchers focus on their specific area, knowing little about its adjacent fields. As an integrated organism, human body consists of different systems that are mutual restraint. The control of the whole body cannot be viewed as sum of controlling different parts. In order to comprehensively regulate the human body, not only the details of parts have to be known, but also the relations between parts have to be regulated. ${ }^{1}$

Today, most patients shun modern medicine's localized treatment and the side effects of chemical drugs. It follows that the development of modern medicine directed by reductionist approach based on feature analysis has come to a dead end. Modern medicine's development and advancement call for the birth of new concepts and methods.

In recent decades, the change in scientific concept brought forth by complexity science affects all scientific fields. A holistic approach gradually replaces the analytical approach to study complex systems such as: human bodies, societies, and ecosystems. This becomes a major method in scientific research. The uses of metaphors and analogies that were considered 'pseudo-science' are now deployed 
to build holistic models in researches. Different from the past theory of entity structure, holistic model describes the regularity and state of research object in an abstractive way. Be it the famous models Sandpile, or Bird Swarm, they are analogous to TCM's theory of visceral manifestation, and identification of defensive-Qi-nutrientblood. It cannot be simply identified that TCM and complexity science are one, but it is clear that the method used in the two systems are of the same strain. ${ }^{2}$

For the past few hundred years, development of western medicine and western science run on parallel tracks. The methods and techniques in science are quickly introduced into the sphere of medicine. They deepen and widen the research work on body and diseases. Nowadays, science research methodology shifts from analytical to holistic approach. In medical field, researchers also come to realize the shortcoming and limitations of their research methodology. They suggest the introduction of functional holistic approach in their research methodology and to establish the idealism of functional holistic medicine. Are we not backward, when in the close encounter of specific cases, viewing the epidemic with spectacles that handed down 300hundred years ago? Obviously, modern medicine severely lags behind the development and advancement of modern science!

Misplaced Belief Solely rely on finding the right vaccine. Virus causes epidemic. Naturally, modern medicine deems it fit to combat the epidemic by finding a way to eradicate or suppress the virus. The focus is directed to finding the right vaccine that may put the whole issue to an end! But there is no safe anti-virus vaccine in the near future.

At present, the study of virus is to isolate it first, then develop drug or vaccine that can kill or inactivated the virus.

\section{Challenges to find the right vaccine}

i. The mutation speed of virus is extremely fast. New types of virus appear before the vaccines are introduced.

ii. Drugs that kill virus in vitro experiments have side effects when tested on human body. It is highly probable that sequelae caused by unsuitable treatment may occur as it was in SARS.

iii. Vaccine that is proved to be safe in clinically trial when put into massive application for treatment or prevention may inevitably yield exceptional cases such as infections and/or complications.

A surprising discovery has been reported in the article "How Ebola Kills You: It's Not The Virus" by Michaeleen Doucleff, a journalist from NPR, on 26 August 2015 that the killer of epidemics is the immune system in our body rather than the Ebola virus. It is the secondary injury caused by response of immune cells that kill the patients.

\section{The following points cannot be ignored when studying the roles of virus during pathogenic process}

i. In the experience of fighting other virus, a healthy person who is found to have carried the virus is called 'health-virus carrier'.

ii. The pathogenic virus can be found from the blood of recovered patients but this virus does not cause illness because the immunity to the virus has been produced in the immune system.

iii. In the process of diseases evolving in human body caused by other viruses, not all harms are caused by the direct attack by virus. The secondary injury is a result of causality chain triggered by the virus.
Obviously, it cannot be concluded that killing virus cure all viral diseases, or that the patient cannot be cured if the virus is not killed.

Thus, it is concluded that the virus is not a decisive factor in the evolving process of viral diseases. Studying virus is not the only way to solve the epidemics. An alternative solution can be found if our attention is directed at the changes and causality inside the infected body.

In the experience of fighting against epidemics, the responses of the body to different viruses in various patients are similar. For example, the clinical symptoms of Ebola at the first stage, such as high fever, headache, muscle and joint pain, and throat pain, are similar to SARS, H3N2. Initial symptoms of bacterial infection are identical.

This means, we will focus our attention on treating virus' symptoms rather than on the patient's physical reactive states to the virus and ignore its mutation or type of virus. So doing, we do not need to find out what type of virus that causes the symptom, whether it caused by virus or bacteria, insofar its harm is stopped and the secondary injury terminated.

It is analogous when a boulder blocks a road. We can always spend time and labour to find out: Where does the boulder come from? Does it fall from the sky? Does it fall from a slope? Fall off from a truck? Does someone place the boulder to cause traffic jam? Of course, the simplest way to solve the immediate problem is to deploy a heavyduty forklift to remove the boulder. Move the boulder away from the main road and let normal traffic resume. And the problem is solved!

The last approach is obviously the simplest and most effective. Direct the treatment of contagious diseases on the reactive states of the patient rather than on the secondary injuries is the key emphasis for TCM practitioners when come to close encounter with epidemics.

As pathogens, virus harms human bodies by interacting with body cells. Pathogens live in the body, becoming an integrated system with human body. The study on holistic system using complexity science is to study the state of changes caused by pathogens. Accordingly, state of changes is interpreted as signs and symptoms that a practitioner may consider as controllable factors, hence, appropriate treatment or herbal drugs can be applied.

In TCM, the pathogenic factors of contagious diseases are classified according to the responses of the human body. Based on the theories of wind, cold, heat, damp, dry, and fire, pathogenic types are categorized as wind-heat, wind-cold, damp-heat etc. This clearly indicates the state of changes of human body. Herbs that can eliminate the signs and symptoms of a certain state thus obviously suppress the pathogens that cause the diseases.

Obviously, considering the state of changes as controllable factors, the methods of treatment and the herbal prescriptions so improvised can be deployed to improve and to eliminate the harm caused by pathogens. If the pathogens are not eradicated, they surely can cause damages and the body's state of changes will deteriorate and the diseases so caused may be beyond repair.

Treating the state of changes as controllable factors will yield an effective mean to eradicate or deactivate the pathogens. ${ }^{3}$

So doing, a holistic model constructed on the basis of holistic medicine is used as a reference to show the state of changes caused by pathogens. The holistic model is macroscopic. The change of states caused by pathogen or interferon on human body (inclusive treatment effects and side effects) may be stable with reference to time and 
space. With such a holistic model and the state of changes as data for reference, the practitioner can optimize treatment process and the use of herbal drugs. This may finally lead to the entire eradication of the cause of disease and repair any possible damages to the human body without adverse side effects that some other treatment plan may bring about.

From the standpoint of modern medicine, we can hardly conclude the research that centers on pathogens is science and the research that centers on the state of changes caused by pathogens is pseudoscience. In modern medicine, there is no system to give a holistic view to describe the state of changes of the human body. Not to mention there is no study or research on the holistic response of the human body.

In the era of complexity science, the model describing complex system is used to study the state of changes under the interference from outside. This model has been widely used in the field of natural science and sociology. It gradually becomes a major method in modern scientific researches.

To introduce complexity science to modern medicine and to build a dynamic model of human body to study the state of changes by the pathogens bring an alternative way to discover the treatments for different pathological states and control the development of diseases. This will be the contribution of natural science's most powerful weapon in the 21 st century to help us and bring about new hope to eradicate complex contagious epidemics.

The medical system describes and control human body states from a holistic view. So far, modern medicine builds its theory on the physiological structure of the human body. TCM, in line with complexity science, builds a holistic human model by ways of analogies and parables (TCM describes it as 'corresponding and analogous'). TCM practitioners understand the stages of an illness base on the holistic view of the human body. Basically, TCM is a medical knowledge focuses on the holistic functional mode of the human body. It embraces modern medicine's basic features on 'functional holistic medicine'.

TCM records a patient's signs and symptoms in a holistic manner. When compare with modern medicine's objective tests and examinations, TCM is undoubtedly, too simple and sometimes inaccurate. So, how does TCM depict human body in a holistic manner?

Influenza shows the signs and symptoms of: fever, headache, muscle pain, sore throat. TCM describe this as the state of 'heat into qi phase', and excessive heat in gastrointestinal. Of course, we can also introduce certain objective examination standard to ascertain the signs and symptoms in a holistic state.

Surely enough, there will be no doubts or questions asked when a patient's signs and symptoms are relieved or conditions improved with the use of TCM methods and herbs. Obviously, viewing these signs and symptoms as a 'barometer' unveiling a patient's body condition may correspond to his internal states. So, using these indications to depict body status as 'observable data' and 'controllable data' may lead to a holistic manageable status of the body condition.

Using objective examination indicators based on signs and symptoms to accurately describe the holistic state of changes caused by illness are introduced, a new system outside of modern medicine is thus established. It forms the basis to deploy new drugs and treatment focusing on the holistic functional states of the human body.
Therefore, under the current framework of modern medicine, a new medical system, using holistic model to describe the state of changes of body during diseases, can be derived.

Concept of organic wholeness is the main characteristics of the theoretic system of TCM that evolved from a long history of combating contagious epidemics. Treatment based on syndrome differentiation includes main symptoms, therapeutic methods, herbal prescription, and herbal modification. This system addresses the different pathological states of the epidemics including: pneumonia, mental disorders, heart, and kidney damages.

Based on the model of organic wholeness as in TCM, a holistic control system to treat the different stages of Ebola epidemic can be incorporated into modern medicine. This may lead to the development of a system in modern medicine that can treat and address the different stages of influenza.

Does it really affect the evidence-based modern medicine when TCM's concept of organic wholeness and treatment based on syndrome differentiation are introduced or incorporated?

In fact, theoretical physics, a forefront science progressing to this modern era, reflects images of certain ancient oriental philosophy: Bootstrapping in quantum physics contains Buddhism's themes of 'my heart is the universe' and 'mass and energy are the same', The Space-time Continuum in Einstein's work and Hawking's 'On Quntum Physics' clearly show that today's theoretical physicists share the same view about the universe as ancient oriental philosophers

As J.R. Oppenheimer, the father of the Atomic Bomb, puts it "The common concepts of human knowledge represented in atomic physics...the innate characters are not unfamiliar and are not completely new to us. They exist in our culture. They form the core theories of Buddhism and Hinduism. All we need do with these ancient wisdom are to discover, to promote, and to fine-tune."

Fritjof Capra, on the issues of science exploration and Eastern philosophy, thus related in his book 'The Tao of Physics': "It is interesting to follow the evolution of Western science along its spiral path, starting from the mystical philosophies of the early Greeks, rising and unfolding in an impressive development of intellectual thought that increasingly turned away from its mystical origins to develop a world view which is in sharp contrast to that of the Far East. In its most recent stages, Western science is finally overcoming this view and coming back to those of the early Greek and the Eastern philosophies. This time, however, it is not only based on intuition, but also on experiments of great precision and sophistication, and on a rigorous and consistent mathematical formalism. ${ }^{4}$

Likewise, we need not worry about the view that modern medicine is "coming back" to traditional medicine. And there should be no worry about the process of integrating the experience and treatment methods of TCM into modern medicine. It is because the coming back builds upon strict scientific experiments and theory models with basis of verification.

Thus, a new modern medical system of state, integrating TCM syndrome differentiation and organic wholeness, can be established according to the idea and methods of complexity science. With this new system and model analysis, physicians may timely control the changes and development of the dynamic virus disease. So doing, physicians may devise treatment method timely and pave a victorious road to combat epidemics. ${ }^{5}$ 
Traditional Chinese Medicine: the successful demonstration of controlling diseases based on body states Indeed, SARS, bird flu of yesterday, or H1N1, Ebola of today, TCM literatures showed that there were epidemics ("Wen Yi") in the past. It happened many times. TCM literatures recorded that there were principles of diagnosis and treatment of epidemics based on the six meridians, the qi, the blood and body fluid, and the triple energizer (Sanjiao).Throughout the past 1,500years, Chinese paid the price of numerous lives to establish a TCM system to combat epidemics.

Zhang Zhongjing (150-219), a famous physician in Han Dynasty and one of the most eminent Chinese physicians in Chinese history, in the preface of his book, so stated "My clan once had a huge population. There were more than two hundred people. Since the first year of Jianan, in less than ten years, two-thirds of my people died. Seven out of ten died of typhoid. I was in deep regrets for the quick decline of the clan and the loss of population. In the midst of deep sorrow and remorse for not able to save lives, I studied diligently, reviewed old medical scrolls such as: Plain Questions, Nine Chapters, 81 Questions, Treatise on Yin and Yang, Tailu Medical Herbs. And I reexamined all herbal formulae. Finally, I came up with this book titled 'A Treatise on Febrile Diseases'. We might not be able to cure all kinds of epidemics. But, with the help of this Treatise, physicians could follow my theory to follow the syndromes to identify the cause of the epidemic. Thus, the problems of typhoid could be solved."

Towards the end of Ming Dynasty and at the beginning of Qing Dynasty, the epidemic occurred intermittently. During the period 1408-1643, there were 19 epidemics. Wu Youxing's book 'On Epidemics', published in 1642, contained the following passage: "... Of 100 families in a locality, there was no survivor. A family of several scores left no survivor!"

Later on, physicians like Ye Tianshi, Wu Jutong, Xue Shengbai, Wu Youke, and Wang Mengyin etc. developed a system within TCM fundamentals to treat the different stages of an epidemic. It included: clinical study, theory, diagnosis, treatment, and herbal prescriptions. The system was proven effective in the combat of epidemics in the years that followed. No epidemics were out of its scope of treatment. Accurate diagnosis coupled with the right herbs, TCM could bring about desirable effects.

In recent years, the status of the booming modern medicine took the lead and surpassed that of TCM. People gradually forgot all about TCM. In particular, TCM is out of the scene when the treatments of acute and contagious diseases are concerned. In the name of science, acute contagious diseases are packaged under terms like bacteria, and virus etc. Hence, the over 1000 years of combat and struggle in TCM looked unrelated and strange. As if there were no epidemics in China, and Chinese people never encountered highly contagious diseases before! Things come to mind are viruses, bacteria, antibiotics, and interferon. TCM is forgotten entirety. In fact, in the modest theory of TCM, there exists a theory and system that consist of treating contagious diseases in different stages: prevention, initial treatment, treatment of compound illness, acute rescue, treatment of side effects, and recovery.

In TCM's theoretical system, regardless of the influenza virus variation, the influenza virus is the equivalent of TCM wind-cold, wind-heat, and damp heat. TCM recorded pathological development of epidemics, seasonal febrile diseases with detailed description of the different stages, patients' state of changes, and detailed records of diagnosis, solutions, and medication. These are our ancestors' treasure trove in combating epidemics and contagious diseases that is similar to today's Ebola. There are a lot of TCM records pinpoint to the effectiveness of combating and healing epidemics. But with modern medicine on the rise, TCM is viewed upon as 'pseudo-science' and is sealed with dust amidst the garbage of science!

In recent decades, the concept of science gradually changes from analysis to synthesis. As complex objective research methods become mature, in response to viral or infectious diseases, the mainstream analysis methodology still leads western medical science. In the face of a new epidemic, mankind turns to the incompetent western medical science for cure. During the SARS epidemic in 2003, the whole world witnessed there were great potentials that TCM could treat contagious diseases.

Body Status Medical: to start the precision medical treatment era for viral infectious diseases In recent years, from alternative medicine, translational medicine to evidence-based medicine, modern medicine has gradually turned to the outside of mainstream medical system to explore the medical future. The success of the human genome project, especially, the gene mutation got significant discovery of a more accurate grasp of the individual states in disease. Modern medicine starts moving towards precision medicine era. American scientific community first proposed the precision medicine program. It marks an end to the disease medical domination era of modern medicine. With the aid of the latest achievements of the genome, proteomics, metabolomics and other omics research, a personalized human disease states description system can be establish, it is the beginning of the body status medical era.

The short-term goal of precision medicine is the precision of cancer treatment. Cancer and viral infectious disease are two extremes. Cancer begins with cell mutation and gradually expanding its scope and impact of erosion, then affecting the whole body finally. Infectious disease caused by virus is a systemic disease right at the beginning and develop rapidly. Both of the two extreme diseases are worlds apart on the requirements of body states description system integrity. In other words, for the treatment of cancer, it does not require a holistic personalized description of the human body pathological status. As long as a more important issue of cancer development and treatment was found, or making a specific cancer therapeutic drug more precisely applicable to the personalized body status, then it will be a significant progress of the treatment to cancer. For viral infectious disease that affecting the whole body, in order to grasp the personalized body status, there must be a complete body states description system.

The US President Barack Obama announced in the 2015 State of the Union, to launch the Million Volunteers Plan in the 2016 fiscal year. In additional to disease medical, it is the first attempt of a systematic and comprehensive body states description system establishment. However, to establish a complete body states description system in genes, proteins, metabolites, and other molecular level, are worthy of its feasibility study.

The human genome sequencing found hundreds of millions of DNA mutations. Today biologists have realized that, in most cases, to determine the pathological states of a body, the role of genes is not decisive. For proteomics, metabolomics indicators are also of great significance. The current study shows that the proteomics and metabolite abnormal group are much more massive than the number of genomes. Of course, the analysis of these data required big data technology, but a lot of the information are in dynamic changing. With such a huge number of indicators, how to instantly acquire and how 
to monitor them, are unthinkable questions. Thus, there are scientists questioned the feasibility and practical significance of precision medicine to establish the body states description system eventually. ${ }^{6}$

We talked about earlier that the contagious and infectious diseases differentiation system of TCM, is the body states description system of systemic disease at the holistic level. It is the same as precision medicine introducing genome and proteome research achievements, to achieve the individual tumor diagnosis and precise treatment. To introduce the syndrome differentiation and treatment system of TCM, keep it precision with the help of modern scientific testing techniques, artificial intelligence and big data analysis techniques. ${ }^{7,8}$ Achieve precise identification and precise treatment for individual body states during the process of viral infectious disease. It is the shortcut for modern medicine dealing with viral infectious disease towards precision medicine era.

\section{Conclusion}

The soaring of complexity science in the field of natural science brings about a revolutionary change in the concept of science. It greatly expands our horizons. Today, facing powerlessly against viral disease, western medicine may, like western natural science, forsake its belief that analytical method is the only scientific method and to adopt the new ideas and methods of complexity science in dealing with complex systems. To establish and adopt the body states description system, just like the precision medicine research on tumor using gene variation studies, then it is expected to open a new window to eradicate epidemics. Hence, for thousands years of clinical practice, TCM established the Syndrome Differentiation and Treatment System. With the ways and means of modern technology to make it more precision, it is the best way to achieve this goal.

\section{Conflicts of interest}

Author declares there are no conflicts of interest.

\section{Funding}

None.

\section{References}

1. Bing Yuan. Traditional Chinese Medicine Modern Methods. China, Wuhan: Hubei Science and Technology Press; 1986. pp. 104-105.

2. Bing Yuan. Holistic Medicine based on the Body Constitutional and Functional Status. Journal of Traditional Chinese Medical. 2012;33(8A):1-3.

3. Bing Yuan. Establishment of Chinese Medicine Research Methods in line with Traditional Chinese Medicine. Chinese Journal of Basic Medicine in Traditional Chinese Medicine. 2000;6(11):34-36.

4. Geng Guan. Modern Physics and Eastern Mysticism. China: Sichuan People's Publishing House; 984. p. 5-7.

5. Bing Yuan. Precision Medicine: Towards Complexity Science Age. Chinese Journal of Integrative Medicine. 2016;22(4):251-257.

6. Bing Yuan. The way of Japanese Kampo Medicine: from the trend of medical development perspective. Guiding Journal of Traditional Chinese Medicine. 2016;36(12A):5-7.

7. Bing Yuan. Establishment of Precise States Description System: the Route that brings TCM to Precision Medicine. Journal of Beijing University of Traditional Chinese Medicine. 2016;39(3):186-190.

8. Bing Yuan. Establishment of Precise States Control System: the Route that brings TCM to Precision Medicine. Journal of Beijing University of Traditional Chinese Medicine. 2016;39(4):211-214.

\section{Acknowledgments}

None. 